\title{
Take a load off: Unloading and reperfusion
}

\author{
Matthew L. Goodwin, MD, and Craig H. Selzman, MD
}

Feature Editor Note-In this issue of the Journal, Drs Goodwin and Selzman have provided a state-of-the-art editorial on the use of mechanical circulatory support as primary therapy to unload the left ventricle as a treatment for acute myocardial infarction. The treatment of acute myocardial infarction has undergone major and significant evolution over the previous decades, from emphasis on primary surgical revascularization, to thrombolytic therapies, to the now-established practice of early reperfusion with percutaneous coronary intervention (PCI). Despite early PCI, significant degrees of myocardium are not salvaged by early reperfusion strategies. With the advent of new and readily available percutaneous left ventricle assist device technologies, the use of mechanical circulatory support to unload the left ventricle as primary therapy to reduce reperfusion injury and infarct size has regained significant interest. This new concept of "door-to-unload" time, which complements the paradigm of "door-to-balloon" time with PCI, has validated the findings of a number of previous historical studies on this topic of ventricular unloading to reduce ischemic reperfusion injury and limit myocardial infarct size. As Drs Goodwin and Selzman review, recent clinical studies have provided evidence of feasibility of this approach, and basic science and translational studies have provided strong biological evidence for the rationale for this treatment paradigm. Reducing wall stress and the inflammatory environment of the ischemic myocardium with mechanical unloading before establishing reperfusion with PCI may ultimately salvage more ischemic myocardium from infarction. The totality of data to date provides intriguing and compelling evidence to suggest this approach warrants further clinical investigation.

\section{Francis D. Pagani, MD, PhD}

Although it remains a leading cause of morbidity and mortality among adults in the United States, the incidence of acute myocardial infarction (AMI) and subsequent inhospital mortality has declined significantly. ${ }^{1}$ Nevertheless,

From the Division of Cardiothoracic Surgery, University of Utah, Salt Lake City, Utah.

Received for publication May 10, 2020; revisions received June 30, 2020; accepted for publication July 8, 2020; available ahead of print July 25, 2020.

Address for reprints: Craig H. Selzman, MD, Division of Cardiothoracic Surgery, Department of Surgery, University of Utah SOM, 1300 North Medical Dr, 3B-

110, Salt Lake City, UT 84132 (E-mail: craig.selzman@hsc.utah.edu).

J Thorac Cardiovasc Surg 2021;161:2046-50

$0022-5223 / \$ 36.00$

Copyright (c) 2020 by The American Association for Thoracic Surgery

https://doi.org/10.1016/j.jtcvs.2020.07.078

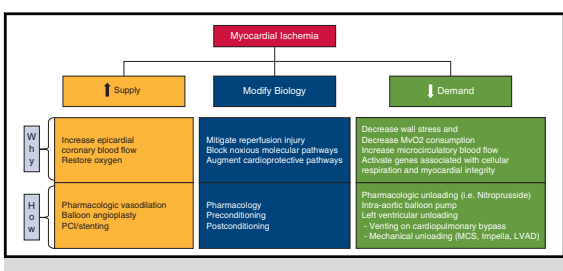

Parallel and companion strategies for effectively treating myocardial ischemic injury.

CENTRAL MESSAGE

Early left ventricular unloading, not just restoration of coronary blood flow, has emerged as a targeted therapy to limit ischemia-reperfusion injury in myocardial infarction.

The Invited Expert Opinion provides a perspective on the following paper: Circulation. 2019 Jan 15;139(3):337-346. TIONAHA.118.038269.

See Commentaries on pages 2051, 2052, and 2054 .

despite the speed and efficiency of reperfusion with contemporary percutaneous interventions, there is an increasing prevalence and mortality impact of heart failure in these patients. ${ }^{2-4}$ Even with early reperfusion, between $20 \%$ and $40 \%$ of the heart has residual infarction, and every $5 \%$ increase in infarct size is associated with nearly a $20 \%$ increase in hospitalizations and mortality at 1 year. ${ }^{5}$ Guidelines promoting door-to-balloon times, early revascularization, and post-myocardial infarction care are well established. ${ }^{6}$ Yet, the current paradigm still allows for significant myocardial damage with both short- and long-term implications. Hence, mitigating myocardial ischemia-reperfusion injury (IRI) remains an imposing clinical challenge.

Myocardial reperfusion injury was first described in by Jennings and colleagues ${ }^{7}$ after temporary coronary occlusion in dogs. Restoration of blood flow to ischemic myocardium is an intuitive and necessary treatment for an occluded coronary artery. The subsequent injury with reperfusion has 
fed a large body of research for more than 60 years-not just with regards to the physiology but the identification of biologic targets that could be modified to impact this phenomenon. ${ }^{8}$ A number of preclinical studies targeting the molecular pathways of IRI have shown promise but ultimately failed to show meaningful improvement in clinical outcomes in myocardial infarction in people. ${ }^{9,10}$ Similarly, newer cardioprotective interventions, like ischemic pre- and postconditioning among others, employed before or during percutaneous coronary intervention (PCI) have shown modest improvements in infarct size or ventricular function but lack clinical outcomes benefit in the literature to date. ${ }^{10}$ Therefore, focus still remains on finding effective biologic therapy for preventing reperfusion injury and improving clinical outcomes in patients with AMI. ${ }^{4}$

\section{DOOR-TO-UNLOADING}

Ventricular unloading, reperfusion, and cardiac function are concepts with which cardiac surgeons are well acquainted, as these principles have guided the development of current cardiac surgery practices, cardiopulmonary bypass, and cardioprotective strategies in the operating room. Recently, ventricular unloading has emerged as a cardioprotective strategy aimed to augment or mitigate IRI in AMI. In vivo and ex vivo studies dating back more than 40 years have repeatedly shown that ventricular unloading improves cardiac function post-AMI. ${ }^{11}$ Translation of these concepts into current clinical practice has been made possible with new percutaneous ventricular assist devices, including the Impella platform (Abiomed, Inc, Danvers, Mass) and the TandemHeart (LivaNova, Mirandola, Italy). A novel concept focusing on ventricular unloading as the initial intervention in AMI before reperfusion, termed door-to-unload (DTU), appears to have benefit in preclinical studies and recently was the targeted therapy in a feasibility and safety trial in humans. ${ }^{12}$

Multiple experimental models have shown that mechanical unloading of the left ventricle, in comparison with reperfusion alone, limits IRI and reduces myocardial infarct size. ${ }^{13,14}$ Ventricular unloading serves to decrease myocardial oxygen demand by decreasing wall stress, enhancing flow in the microcirculation, and releasing a number of circulating factors that favorably impact cardiac remodeling. For this to work, however, it takes some time. Kapur and colleagues ${ }^{12}$ demonstrated in an elegant porcine model that the positive effects of unloading required 30 minutes (compared with 15 minutes) of unloading before reperfusion. Compared with primary reperfusion, these unloaded hearts had increased expression of genes associated with cellular respiration and mitochondrial integrity as well as decreased levels of proteases. ${ }^{15}$

Kapur and colleagues ${ }^{12}$ have recently extended their animal models to investigate the impact of ventricular unloading before reperfusion in patients with AMI without cardiogenic shock. In this prospective, multicenter, randomized pilot study, all patients with anterior STelevation myocardial infarction received initial ventricular unloading with the Impella CP system (Abiomed Inc). Patients were randomized to left ventricular unloading followed by immediate reperfusion or unloading with a 30-minute delay to reperfusion. The 30-minute time delay was selected based on preclinical data showing that unloading and delaying reperfusion for 30 minutes activates cardioprotective signaling, which reduces myocardial injury and promotes myocardial recovery after reperfusion. The primary safety end point was a composite of major adverse cardiovascular and cerebrovascular events at 30 days. The authors assessed the infarct size as a percentage of total left ventricular mass using cardiac magnetic resonance imaging at 30 days as the primary efficacy end point. Secondary outcomes included all-cause mortality, hemolysis, acute renal dysfunction, rehospitalization for heart failure, ventricular arrhythmias, left ventricular thrombus, bleeding, and minor vascular events.

A total of 50 patients were enrolled in the trial. Major adverse cardiovascular and cerebrovascular events at 30 days was not significantly different between the unloading with delayed reperfusion and immediate reperfusion cohorts (3/25 [12\%] vs $2 / 25$ [8\%], $P=.99$, respectively). In addition, delayed reperfusion did not affect 30-day mean infarct size as a percentage of left ventricular mass when assessed with cardiac magnetic resonance imaging. Furthermore, there was no difference in any of the secondary outcomes between groups. With the 30-minute delay designed into the study protocol, the door-to-balloon time for the delayed reperfusion group was greater than $90 \mathrm{mi}-$ nutes (mean 97 minutes). The study deserves credit, as it was the first human experience with ventricular unloading and intentional delayed reperfusion in AMI. It showed that mechanical unloading can proceed in a timely and safe fashion after presentation with chest pain in AMI.

There are a number of important limitations in this study. Most importantly, there was not a standard-of-care control group in which coronary perfusion is restored without mechanical unloading. Although the size of the infarcts based on presentation ST-elevation was mostly large, these were not patients in cardiogenic shock. In fact, mean arterial pressures were well over $100 \mathrm{~mm} \mathrm{Hg}$ in both groups. We normally think of placing mechanical devices in patients with poor hemodynamics; will the community put an expensive device that has potential for vascular or valvular complications in patients who are not presenting in shock or with very small infarcts? Quite possibly, infarct sizes with a door-to-balloon strategy would reveal similar results. Another issue that is difficult to test in humans is the length of ischemia. The patients in this study had chest pain between 1 and 5 hours. In experimental models, one can be very precise with timing. Is there a point of ischemic time 
whereby delaying reperfusion actually makes the infarct worse, despite unloading? Ultimately, however, the safety demonstrated in this pilot study enables a phase III trial comparing unloading with primary PCI without unloading to be undertaken (STEMI-DTU trial, NCT: 03947619).

\section{UNLOADING AND REPERFUSION}

The majority of literature related to unloading is related to patients in the throes of cardiogenic shock, protected percutaneous interventions or ablations, and postcardiotomy shock. There is a dearth of literature regarding the impact of preoperative unloading before coronary artery bypass surgery, especially with advent of the sleeker and more robust percutaneous devices and extracorporeal membrane oxygenation. Although most centers have used these approaches in lieu of emergent salvage coronary artery bypass grafting, we do this mostly by our clinical (and anecdotal) intuition rather than following the path of a welldesigned study in this arena. The intra-aortic balloon pump provided much of our current teaching that highrisk patients requiring coronary artery bypass surgery (acute myocardial infarction, poor left ventricular function, complications of myocardial infarction) are best served with its preoperative placement. Studies back in the 1970s identified the impact on unloading, metabolism, and survival with this preoperative approach. ${ }^{16}$ To date, use of the more-sophisticated percutaneous heart pumps are mostly limited to case reports. Whether routine use of these more expensive pumps as prophylaxis remains to be seen and studied.

Although the ischemia and reperfusion stress during cardiac surgery is distinctly different from that of an AMI, the door-to-unloading concept uses similar cardioprotective mechanisms as ventricular unloading in the operating room. ${ }^{17}$ Indeed, there are considerable overlaps in the investigation of cardioprotective strategies in myocardial infarction and cardiac surgery. Early studies on unloading for myocardial infarction used a cardiopulmonary bypass circuit to empty the ventricle. Studies in the 1980s showed that unloading via left atrial to femoral artery bypass during occlusion significantly decreased infarct size even without reperfusion. ${ }^{8,18}$ Furthermore, using left-heart bypass, others showed similar reduction in infarct size with unloading during the reperfusion phase alone. ${ }^{19}$ Contemporary studies in cardiac surgery showed the negative effects of ventricular distention during postischemic reperfusion. In an animal experiment using a cardiopulmonary bypass circuit, left ventricular vent, and intraventricular balloon to control left ventricular end-diastolic pressure, Lucas and colleagues ${ }^{20}$ showed the harmful effects of even mild distention during early reperfusion after ischemic arrest. The poor ventricular function postarrest in the distended hearts was attributed to increased transmural wall tension and decreased subendocardial blood flow compared with the control, nondistended hearts. In the distended hearts, the increased wall tension led to subendocardial ischemia, as evidenced by increased intramyocardial $\mathrm{CO}_{2}$, lactate, and myocardial oxygen consumption. ${ }^{20}$

Previous studies such as these lay the foundation for many current cardiac surgery practices. Reperfusion injury can manifest as arrhythmia, myocardial stunning, low cardiac output, and perioperative myocardial infraction. ${ }^{17}$ In patients suffering a perioperative death after coronary artery bypass surgery, histologic evidence of IRI is present on autopsy in $25 \%$ to $45 \%$ of patients. ${ }^{21,22}$ In addition, elevated cardiac biomarkers postoperatively have been associated adverse events and mortality. ${ }^{23}$ Recognizing and avoiding ventricular distention is dogma to our profession. Planning for an operation includes assessment of valvular and ventricular function as well as devising strategies for cannulation, temperature management, delivery of cardioplegia solution, venting, and weaning from cardiopulmonary bypass. These maneuvers are designed, in part, to limit myocardial oxygen consumption and ventricular work but also to regulate the deleterious effects of reperfusion injury that is exacerbated by ventricular distention.

\section{CONCLUSIONS}

The increasing incidence of heart failure in patients post-AMI illustrates the limitations of current therapy for preventing or mitigating IRI. The concept that restoration of epicardial coronary blood flow alone is sufficient to treat this patient is short-sighted. Quite possibly, restoration of blood flow in the face of ventricular distension can actually increase injury as the increased wall stress and inflammatory milieu might contribute to microcirculatory dysfunction (no-reflow phenomenon) and persistent subendocardial ischemia. DTU has emerged as a novel cardioprotective strategy, with a recent pilot study demonstrating safety and feasibility of this technique. What DTU accomplishes before and during reperfusion is the same cardioprotective effects of ventricular unloading and cardiopulmonary bypass in the cardiac operating room, namely: decreased myocardial oxygen consumption, activation of cardioprotective signaling, increased myocardial microvascular perfusion, and hemodynamic stabilization through reperfusion-dependent arrhythmias and myocardial stunning. ${ }^{11}$

Cardiac surgeons should follow the DTU story enthusiastically, as the outcomes and advancement of the science of IRI can advance our own specialty. In addition to unloading, multiple cardioprotective strategies to limit reperfusion injury have been simultaneously investigated as adjuncts to PCI and cardiac surgery. These include ischemic preconditioning, immune system modulation, temperature control, and metabolic additives and antagonist. The information gained from these combined efforts has advanced our 


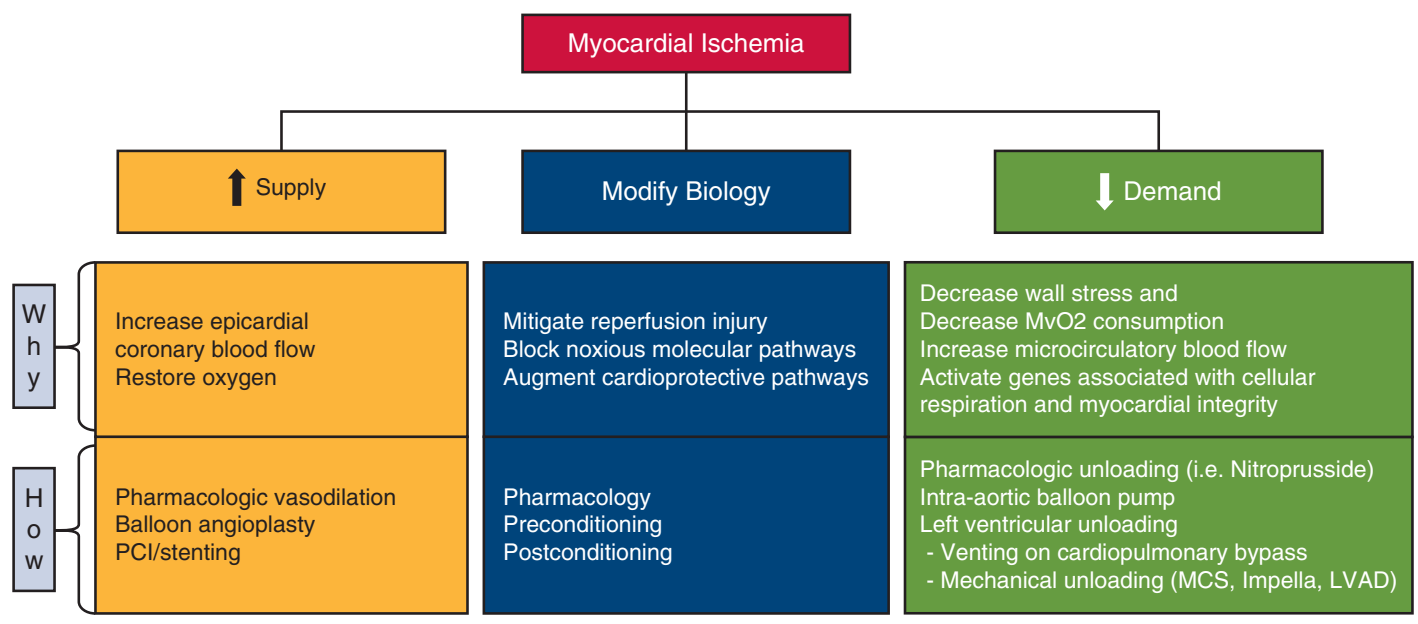

FIGURE 1. Parallel and companion strategies for effectively treating myocardial ischemic injury. $P C I$, Percutaneous coronary intervention; $M V O_{2}$, myocardial oxygen consumption; $M C S$, mechanical circulatory support; LVAD, left ventricular assist device.

own specialty, cardioprotective strategies, and care of our patients.

Will DTU suffer the same fate as other therapies targeting IRI in which encouraging preclinical data were followed by underwhelming clinical outcomes? The study by Kapur and colleagues leaves many questions as to the best application-including, but not limited to, length of time from symptoms, size of infarcts, locations of infarct—of a significant resource that might not be universally available to hospitals and patients. The maxim, "time is muscle" will be difficult to ignore with an intentional, delayed reperfusion without convincing evidence. The theme of investigations in this space, and what has long been noted and used by cardiac surgeons, is that the efficacy of reperfusion therapy is limited in hearts that remain loaded. As depicted in Figure 1, studies done by Kapur and colleagues and others catalyze future research that must integrate the components involved with myocardial IRI: effective management requires more than just restoring epicardial coronary blood flow (supply) but needs to be pursued in companion with decreasing demand (unloading) and modification of the biologic milieu.

\section{Conflict of Interest Statement}

The authors reported no conflicts of interest.

The Journal policy requires editors and reviewers to disclose conflicts of interest and to decline handling or reviewing manuscripts for which they may have a conflict of interest. The editors and reviewers of this article have no conflicts of interest.

\section{References}

1. Benjamin EJ, Muntner P, Alonso A, Bittencourt MS, Callaway CW, Carson AP, et al. Heart disease and stroke statistics-2019 update: a report from the American Heart Association. Circulation. 2019;139:e56-528.
2. Bahit MC, Kochar A, Granger CB. Post-myocardial infarction heart failure. JACC Heart Fail. 2018;6:179-86.

3. Cahill TJ, Kharbanda RK. Heart failure after myocardial infarction in the era of primary percutaneous coronary intervention: mechanisms, incidence and identification of patients at risk. World J Cardiol. 2017;9:407-15.

4. Hausenloy DJ, Yellon DM. Targeting myocardial reperfusion injury-the search continues. N Engl J Med. 2015;373:1073-5.

5. Stone GW, Selker HP, Thiele H, Patel MR, Udelson JE, Ohman EM, et al. Relationship between infarct size and outcomes following primary pci: patient-level analysis from 10 randomized trials. J Am Coll Cardiol. 2016; 67:1674-83.

6. Amsterdam EA, Wenger NK, Brindis RG, Casey DE Jr, Ganiats TG, Holmes DR Jr, et al. 2014 AHA/ACC guideline for the management of patients with non-ST-elevation acute coronary syndromes: executive summary: a report of the American College of Cardiology/American Heart Association task force on practice guidelines. Circulation. 2014;130:2354-94.

7. Jennings RB, Sommers HM, Smyth GA, Flack HA, Linn H. Myocardial necrosis induced by temporary occlusion of a coronary artery in the dog. Arch Pathol. 1960;70:68-78.

8. Acharya D. Unloading and reperfusion in myocardial infarction: a matter of time. Circ Heart Fail. 2020;13:e006718.

9. Bolli R, Becker L, Gross G, Mentzer R Jr, Balshaw D, Lathrop DA, et al. Myocardial protection at a crossroads: the need for translation into clinical therapy. Circ Res. 2004;95:125-34.

10. Yellon DM, Hausenloy DJ. Myocardial reperfusion injury. N Engl J Med. 2007; 357:1121-35.

11. Curran J, Burkhoff D, Kloner RA. Beyond reperfusion: acute ventricular unloading and cardioprotection during myocardial infarction. J Cardiovasc Transl Res. 2019;12:95-106.

12. Kapur NK, Alkhouli MA, DeMartini TJ, Faraz H, George ZH, Goodwin MJ et al. Unloading the left ventricle before reperfusion in patients with anterior ST-segment-elevation myocardial infarction. Circulation. 2019;139: 337-46.

13. Achour H, Boccalandro F, Felli P, Amirian J, Uthman M, Buja M, et al. Mechanical left ventricular unloading prior to reperfusion reduces infarct size in a canine infarction model. Catheter Cardiovasc Interv. 2005;64:182-92.

14. Saku K, Kakino T, Arimura T, Sunagawa G, Nishikawa T, Sakamoto T, et al. Left ventricular mechanical unloading by total support of Impella in myocardial infarction reduces infarct size, preserves left ventricular function, and prevents subsequent heart failure in dogs. Circ Heart Fail. 2018; 11:e004397.

15. Esposito ML, Zhang Y, Qiao X, Reyelt L, Paruchuri V, Schnitzler GR, et al. Left ventricular unloading before reperfusion promotes functional recovery after acute myocardial infarction. J Am Coll Cardiol. 2018;72:501-14.

16. Feola M, Wiener L, Walinsky P, Kasparian H, Duca P, Gottlieb R, et al Improved survival after coronary bypass surgery in patients with poor left 
ventricular function: role of intraaortic balloon counterpulsation. Am J Cardiol. 1977;39:1021-6.

17. Turer AT, Hill JA. Pathogenesis of myocardial ischemia-reperfusion injury and rationale for therapy. Am J Cardiol. 2010;106:360-8.

18. Laschinger JC, Cunningham JN Jr, Catinella FP, Knopp EA, Glassman E, Spencer FC. 'Pulsatile' left atrial-femoral artery bypass. A new method of preventing extension of myocardial infarction. Arch Surg. 1983;118:965-9.

19. Laschinger JC, Grossi EA, Cunningham JN Jr, Krieger KH, Baumann FG, Colvin SB, et al. Adjunctive left ventricular unloading during myocardial reperfusion plays a major role in minimizing myocardial infarct size. J Thorac Cardiovasc Surg. 1985;90:80-5.

20. Lucas SK, Schaff HV, Flaherty JT, Gott VL, Gardner TJ. The harmful effects of ventricular distention during postischemic reperfusion. Ann Thorac Surg. 1981; 32:486-94.
21. Moore GW, Hutchins GM. Coronary artery bypass grafts in 109 autopsied patients. Statistical analysis of graft and anastomosis patency and regional myocardial injury. JAMA. 1981;246:1785-9.

22. Weman SM, Karhunen PJ, Penttila A, Jarvinen AA, Salminen US. Reperfusion injury associated with one-fourth of deaths after coronary artery bypass grafting. Ann Thorac Surg. 2000;70:807-12.

23. Jacobs JP, Alam SS, Owens SL, Parker DM, Rezaee M, Likosky DS, et al. The association between novel biomarkers and 1-year readmission or mortality after cardiac surgery. Ann Thorac Surg. 2018;106:1122-8.

Key Words: myocardial ischemia-reperfusion injury, ventricular unloading, Impella, mechanical circulatory support, myocardial infarction 